\title{
Evaluation of earth rotation parameters from modernized GNSS navigation messages
}

\author{
Peter Steigenberger $^{1}$ (i) $\cdot$ Oliver Montenbruck ${ }^{1}$ (i) $\cdot$ Markus Bradke $^{2}$ (D) Markus Ramatschi $^{2} \cdot$ Uwe Hessels $^{3}$
}

Received: 9 September 2021 / Accepted: 18 January 2022 / Published online: 19 February 2022

(c) The Author(s) 2022

\begin{abstract}
Modernized navigation messages of global navigation satellite systems like GPS CNAV include earth rotation parameters (ERPs), namely the pole coordinates and UT1-UTC $(\triangle \mathrm{UT} 1)$ as well as their rates. Broadcast ERPs are primarily needed for space-borne GNSS applications that require transformations between earth-fixed and inertial reference frames like navigation in earth orbit as well as to the moon. Based on a global tracking network of 23 stations, broadcast ERP values are obtained for the global systems GPS and BeiDou as well as the regional QZSS and IRNSS. Subsequent data sets at daily intervals show polar motion discontinuities of 0.4 to 0.7 mas for GPS, QZSS, and IRNSS, whereas BDS is worse by a factor of about two. Discontinuities in $\triangle \mathrm{UT} 1 \mathrm{range}$ from 0.17 to $0.45 \mathrm{~ms}$. External comparison with the C04 series of the International Earth Rotation and Reference Systems Service results in polar motion RMS differences of 0.3 to 1.0 mas and $\Delta$ UT1 differences of about $0.13 \mathrm{~ms}$ for GPS, QZSS, and IRNSS. Due to less frequent update intervals, BDS performs worse by a factor of $2-4$. In view of the current GNSS-based positioning errors at geostationary or even lunar distances, the accuracy of GPS, QZSS, and IRNSS broadcast ERPs is sufficient to support autonomous spacecraft navigation without the need for external data.
\end{abstract}

Keywords Earth orientation parameters $\cdot$ GNSS $\cdot$ ERP $\cdot$ Polar motion $\cdot$ UT1

\section{Introduction}

The navigation messages of Global Navigation Satellite Systems (GNSSs) provide essential information such as satellite orbit and clock parameters needed for GNSS-based positioning. Modernized navigation messages like the civil navigation message (CNAV) include additional data such as earth rotation parameters (ERPs). The ERPs comprise the pole coordinates $x_{p}$ and $y_{p}$, and the difference $\Delta \mathrm{UT} 1$ between Universal Time 1 (UT1) and Universal Time Coordinated (UTC). The pole coordinates describe the location of the

Peter Steigenberger

peter.steigenberger@dlr.de

1 Deutsches Zentrum für Luft- und Raumfahrt (DLR), German Space Operations Center (GSOC), Münchener Straße 20, 82234 Weßling, Germany

2 Helmholtz-Zentrum Potsdam, Deutsches GeoForschungsZentrum, Telegrafenberg, 14473 Potsdam, Germany

3 Bundesamt für Kartographie und Geodäsie (BKG), Geodätisches Observatorium Wettzell, Sackenrieder Straße 25, 93444 Bad Kötzting, Germany earth rotation axis on the earth's crust. $\Delta \mathrm{UT} 1$ is based on the difference between earth rotation (UT1) and an atomic time scale (UTC).

The ERPs are part of the full set of earth orientation parameters (EOPs) which, in addition, include precession and nutation angles or celestial pole coordinates, and describe the overall transformation between the earth-centered earth-fixed (ECEF) and the earth-centered inertial (ECI) reference frame (Torge and Müller 2012; Capitaine 1986). Only the ERPs are included in the GNSS navigation message as nutation and precession can be described by analytical models with sufficient accuracy over long time scales. Therefore, only these parameters will be considered in the following.

For completeness, it should also be mentioned that the Interface Control Documents (ICDs) of the various GNSSs consistently use the term "EOP" when describing the format and usage of the corresponding parameters, even though the designation "ERP" would actually be more appropriate and consistent with established geodetic terminology. In fact, all currently available broadcast navigation messages are confined to polar motion and $\triangle \mathrm{UT} 1$ data and do not provide additional parameters for the full ECEF/ECI transformation. 


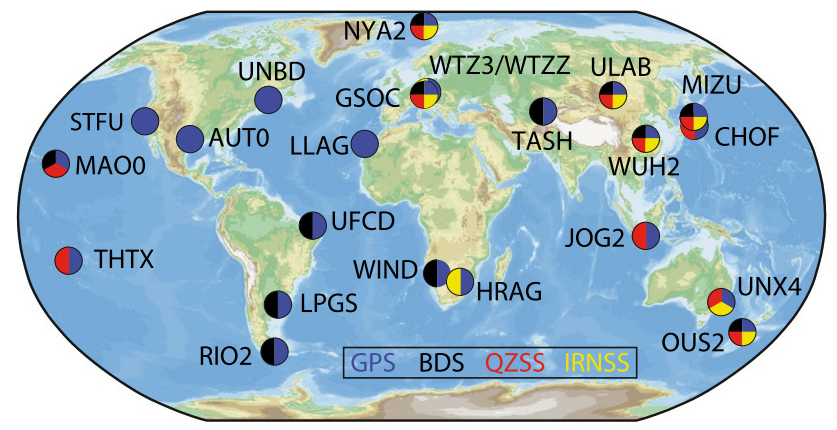

Fig. 1 GNSS tracking stations used for the collection of raw navigation data frames

ERPs are of particular importance for space-borne GNSS applications as orbit computations and predictions are usually performed in an ECI frame whereas the GNSS satellite orbits are given in an ECEF frame. These applications include low earth orbit (LEO) satellites for, e.g., earth observation, navigation of geostationary satellites to their designated orbital slot as well as navigation to and around the moon. ERPs are also required for the numerical propagation of GNSS satellite orbits over extended periods of time. If the ERPs are included in the navigation message, such a propagation can be performed autonomously inside a GNSS receiver without the need for external data (Bar-Sever and Bertiger 2012; Montenbruck et al. 2020).

ERP data are currently transmitted by the United States Global Positioning System (GPS), the Chinese BeiDou Navigation Satellite System (BDS), the Japanese QuasiZenith Satellite System (QZSS), and the Indian Regional Navigation Satellite System (IRNSS). For our study, ERP data were decoded from raw navigation frames provided by 23 globally distributed receivers from two different manufacturers, see Fig. 1, ensuring continuous coverage. The stations are part of the Cooperative Network for GNSS Observations (CONGO, Montenbruck et al. 2011) operated by the German Aerospace Center (DLR) and the German Federal Agency for Cartography and Geodesy (BKG) as well as additional stations contributed by Deutsches GeoForschungsZentrum (GFZ, Ramatschi et al. 2019). For further processing, the extracted ERP data were stored in a draft version of the Receiver Independent Exchange format 4.00 (RINEX, IGS RWG and RTCM 2021). Our analysis covers the time period January 2020 until May 2021.

First, an overview of reference frame transformations and ERPs is given. The broadcast ERP data, including the transmission schemes of the different systems, are discussed in the following section. Finally, the quality of the broadcast ERPs is analyzed by misclosures of consecutive data sets as well as comparisons with an external reference series.

\section{Reference frame transformations and earth rotation parameters}

As a background for better understanding, this section describes the established models for transformations between celestial and terrestrial reference frames and highlights the role of ERPs in this transformation.

\section{Reference frame transformations}

The transformation from the ECEF to the ECI frame is conventionally composed of a sequence of three rotations (Petit and Luzum 2010):

$\mathbf{r}_{\mathrm{ECI}}=\mathbf{Q}(t) \cdot \mathbf{R}(t) \cdot \mathbf{W}(t) \cdot \mathbf{r}_{\mathrm{ECEF}}$

with the earth-centered inertial position vector $\mathbf{r}_{\mathrm{ECI}}$ and the earth-centered earth-fixed position vector $\mathbf{r}_{\mathrm{ECEF}}$. The matrix $\mathbf{Q}$ describes essentially the combined effect of precession and nutation. It depends on the coordinates $\mathrm{X}$ and $\mathrm{Y}$ of the celestial intermediate pole (CIP) in the celestial intermediate reference system (CIRS), which are described by the IAU2006/IAU2000A model (Wallace and Capitaine 2006). The matrix $\mathbf{R}$ is based on the earth rotation angle (ERA), which, by definition, depends on UT1 in a strictly linear manner.

The transformation matrix for polar motion $\mathbf{W}$ is composed of rotation matrices $\mathbf{R}_{i}$ around the axis $i=1,2,3 \mathrm{com}$ puted from the pole coordinates $x_{p}$ and $y_{p}$ and the locator $s^{\prime}$ of the terrestrial intermediate origin (TIO)

$\mathbf{W}=\mathbf{R}_{3}\left(-s^{\prime}\right) \cdot \mathbf{R}_{1}\left(y_{p}\right) \cdot \mathbf{R}_{2}\left(x_{p}\right)$

The legacy reference frame transformation recommended in the IERS Conventions 1996 (McCarthy 1996) is based on the nutation matrix N (IAU1980, Seidelmann 1982), the precession matrix P (IAU1976, Lieske et al. 1977) and Greenwich Apparent Sidereal Time (GAST)

$\mathbf{r}_{\mathrm{ECI}}=\mathbf{P}(t) \cdot \mathbf{N}(t) \cdot \mathbf{R}_{3}(-\mathrm{GAST}) \cdot \mathbf{R}_{2}\left(x_{p}\right) \cdot \mathbf{R}_{1}\left(y_{p}\right) \cdot \mathbf{r}_{\mathrm{ECEF}}$

Other than the ERA, GAST depends on UT1 in a nonlinear manner and involves nutation-related periodic contributions (McCarthy 1996). Whereas this transformation is outdated for high precision applications, it still provides sufficient accuracy when using broadcast ERPs. Compared to the full IERS2010 models, it offers higher efficiency and lower computational effort, in particular, if the number of nutation model coefficients is further reduced (Montenbruck et al. 2020). 


\section{Earth rotation parameters}

Variations in earth rotation are primarily driven by tidal forces of sun and moon as well as mass transport in the earth system. They are dominated by the typical tidal periods of 14 days and one month as well as variations at semiannual, annual, and Chandler periods. The Chandler period is about 435 days and caused by the free oscillation of the earth (Dehant and Mathews 2015). ERPs can be estimated from observations of the space-geodetic techniques Satellite Laser Ranging (SLR, Pearlman et al. 2019), Very Long Baseline Interferometry (VLBI, Schuh and Behrend 2012), Doppler Orbitography integrated by Satellite (DORIS, Gambis 2006), and Global Navigation Satellite Systems (GNSSs). The satellite techniques GNSS, SLR, and DORIS are not able to estimate $\Delta \mathrm{UT} 1$ due to correlations with the right ascension of the ascending node of artificial satellites (Rothacher et al. 1999). However, they are capable of determining the excess length of day ( $\Delta \mathrm{LOD})$ which is related to the time derivative of $\Delta \mathrm{UT} 1$ by

$\Delta \mathrm{LOD}=-\frac{d}{d t}(\mathrm{UT} 1-\mathrm{UTC}) \cdot 86400 \mathrm{~s}=-\Delta \mathrm{UT} 1 \cdot 86400 \mathrm{~s}$

in the absence of leap second changes. ERPs are typically provided as daily estimates of offset and drift at noon or offset values at midnight. However, earth rotation is also affected by subdaily effects primarily introduced by ocean tides. These variations at diurnal and semidiurnal periods can reach up to 1 mas for polar motion and $0.1 \mathrm{~ms}$ for $\Delta \mathrm{UT} 1$, see Fig. 2. The largest constituents for polar motion are the lunar diurnal tide $\mathrm{K}_{1}$ with a period of $23.93 \mathrm{~h}$ and the principal lunar semidiurnal tide $\mathrm{M}_{2}$ with a period of $12.42 \mathrm{~h}$.

For high-precision applications, a model for these subdaily effects has to be added to the daily ERP estimates, which, by convention, are mean values free of subdaily tide contributions. The model recommended by the International Earth Rotation and Reference Systems Service (IERS) can be found in the recent IERS conventions (Petit and Luzum 2010). The complete IERS2010 model consists of 41 diurnal and 30 semidiurnal terms. The superposition of different periods introduces beat periods, the most prominent ones with 14 days, half a year and a year. The beat period of about 14 days can clearly be seen in Fig. 2. If ERPs are estimated at daily intervals, the subdaily ERP variations have to be corrected in the observation model to avoid aliasing effects. As a consequence, the estimated ERPs are free of the subdaily effects, and they have to be added by the user after interpolating or propagating the tabulated ERPs to the desired epoch.

In addition to these subdaily variations, $\Delta \mathrm{UT} 1$ and $\Delta$ LOD are affected by tidal deformations of the earth
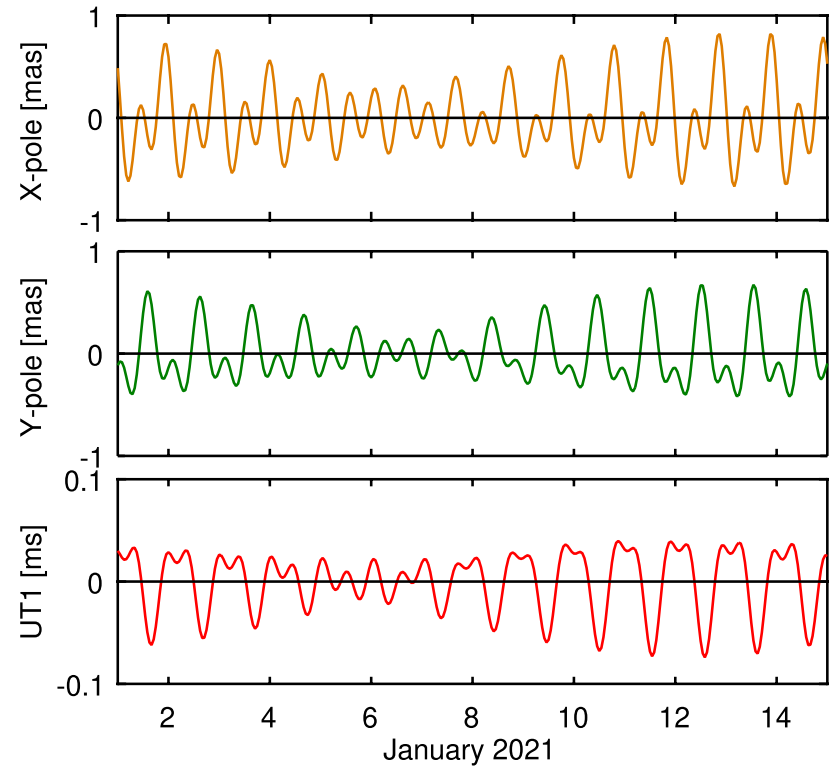

Fig. 2 Subdaily corrections for polar motion and UT1 for the first two weeks of January 2021
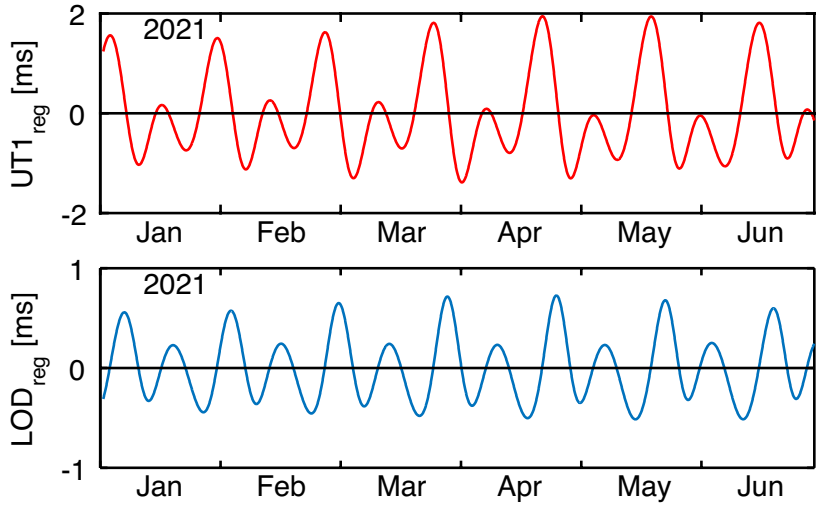

Fig. 3 Corrections due to zonal tides for UT1 and LOD with periods between 5 and 35 days

caused by sun and moon with periods between 5 days and 18.6 years (zonal tides, Yoder et al. 1981). Whereas the linear ERP estimation model sufficiently approximates long-periodic variations, variations with periods up to roughly one month should be considered for best interpolation or extrapolation of tabular ERP data based on a daily epoch grid. Regularized versions of $\Delta \mathrm{UT} 1$ and $\Delta$ LOD in which zonal tides with periods between 5 and 35 days as given in Table 8.1 of Petit and Luzum (2010) have been removed are designated as $\triangle \mathrm{UT} 1 \mathrm{R}$ and $\triangle \mathrm{LODR}$, respectively. A one-year time series of correction terms $\mathrm{UT} 1_{\text {reg }}=\Delta \mathrm{UT} 1-\Delta \mathrm{UT} 1 \mathrm{R}$ and $\mathrm{LOD}_{\text {reg }}=\Delta \mathrm{LOD}-\Delta \mathrm{LODR}$ is given in Fig. 3. This plot is dominated by the largest terms of the 41 constituents, namely the lunisolar 
fortnightly tide $\mathrm{M}_{f}$ with a period of 13.66 days and the lunar monthly tide $\mathrm{M}_{m}$ with a period of 27.56 days.

In the determination of $\Delta \mathrm{UT} 1$, the periodic part $\mathrm{UT} 1_{\text {reg }}$ is removed from the observed $\Delta \mathrm{UT} 1$ to get the smoother quantity $\Delta \mathrm{UT} 1 \mathrm{R}$ to improve the accuracy of linear inter-/ extrapolation. After the parameter estimation, $\mathrm{UT}_{\text {reg }}$ is added back to obtain $\Delta \mathrm{UT} 1$. The same procedure has to be applied when propagating $\Delta \mathrm{UT} 1$ from the data reference epoch $\mathrm{t}_{\mathrm{ERP}}$ to an arbitrary epoch t. As illustrated in Fig. 4, $\Delta \mathrm{UT} 1$ and $\Delta \mathrm{LOD}$ given at $\mathrm{t}_{\mathrm{ERP}}$ are first reduced to $\Delta \mathrm{UT} 1 \mathrm{R}$ and $\triangle \mathrm{LODR}$. Then $\triangle \mathrm{UT} 1 \mathrm{R}$ is propagated to $\mathrm{t}$ with $\Delta \mathrm{LODR}$ and $\mathrm{UT} 1_{\text {reg }}$ at $\mathrm{t}$ is added back to obtain $\Delta \mathrm{UT} 1$ at t. Finally, the subdaily corrections for polar motion and $\Delta \mathrm{UT} 1$ have to be added to get $x_{p}, y_{p}$, and $\Delta \mathrm{UT} 1$ at epoch $\mathrm{t}$.

An overview about the characteristic errors of different commonly used ERP series is given in Table 1. Polar motion and $\triangle \mathrm{UT} 1$ are given in units of milliarcseconds (mas) and milliseconds (ms) corresponding to about 3 and $46 \mathrm{~cm}$ on the earth's surface, respectively. Bulletin A (Wooden and Gambis 2004) is a multi-technique combined EOP series derived from GNSS, SLR, and VLBI observations as well as atmospheric angular momentum (AAM) data obtained from meteorological analysis and forecasts. It includes one year of predictions and is updated on a weekly basis.

IERS C04 (Bizouard et al. 2018) is also a combined multi-technique ERP series but has a higher latency than

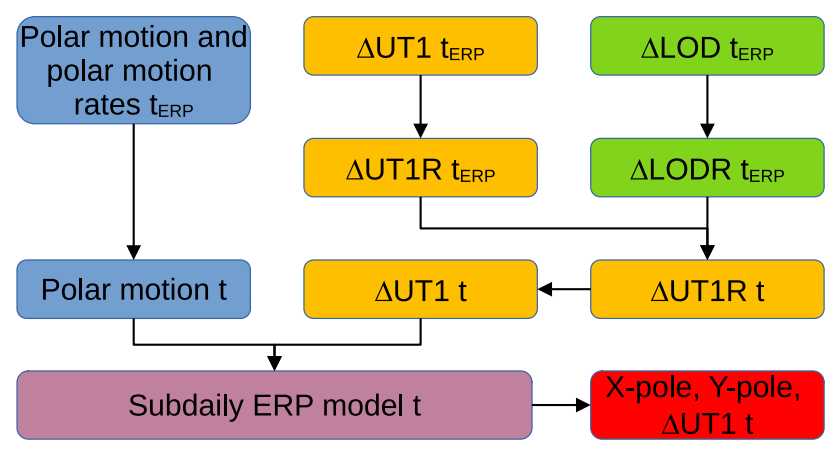

Fig. 4 Schematic overview how to compute ERP values for epoch $t$ from tabulated offsets and rates at $t_{\mathrm{ERP}}$
Bulletin A and does not include predictions. It is based on the combined ERP series of the International GNSS Service (IGS, Johnston et al. 2017), the International VLBI Service (IVS, Nothnagel et al. 2016), the International Laser Ranging Service (ILRS, Pearlman et al. 2019), and the International DORIS Service (IDS, Willis et al. 2015). In addition, input data of individual VLBI analysis centers (ACs) and the ERP data of the International Terrestrial Reference Frame (ITRF) combination are considered. Due to the latencies of the input series, IERS C04 is provided with a delay of one month after the last data point.

The IGS provides ERP series with different latencies obtained from a combination of the results contributed by several ACs. The IGS ultra-rapid ERP series (IGS 2021a) includes one day of observed ERP data as well as one day of predicted ERPs. As GNSSs are not able to determine $\triangle \mathrm{UT} 1$, the ultra-rapid $\triangle \mathrm{UT} 1$ values are derived from Bulletin A. The IGS final combined ERP series (IGS 2021b) has a latency of about two weeks and contains only values obtained from observations. The quality of the IGS final polar motion estimates as well as their error budget, is assessed in detail by Ray et al. (2017).

\section{ERP data in GNSS navigation messages}

ERPs are included in the GPS/QZSS CNAV and CNAV-2, BeiDou CNAV-1, CNAV-2 and CNAV-3, as well as IRNSS NAV messages, see Table 2. For GLONASS, transmission of ERPs is foreseen for the navigation messages of future L1, L2, and L3 code division multiple access (CDMA) signals, but no such data are broadcast at present. For Galileo, no ERP data are included in the current Open Service signals and no plans for future transmissions have been announced so far. Format descriptions and user guidelines are given in the ICDs of the individual systems, also listed in Table 2. Identical ERP parameters and formats are defined across the various navigation message types within each constellation. Furthermore, identical ERP values are provided in the different messages concurrently transmitted by an individual satellite. As such, no distinction is made between ERPs of individual messages
Table 1 Characteristic errors of selected ERP series

\begin{tabular}{llll}
\hline ERP series & $\begin{array}{l}\text { Polar motion } \\
{[\mathrm{mas}]}\end{array}$ & $\Delta \mathrm{UT1}[\mathrm{ms}]$ & Reference \\
\hline Bulletin A, observed & 0.1 & 0.02 & Wooden and Gambis (2004) \\
Bulletin A, 1-day prediction & 0.6 & 0.11 & Wooden and Gambis (2004) \\
Bulletin A, 5-day prediction & 2.6 & 0.42 & Wooden and Gambis (2004) \\
IERS C04, observed & 0.03 & 0.01 & Bizouard et al. (2018) \\
IGS ultra-rapid, observed & 0.02 & - & ultra-rapid versus final \\
IGS ultra-rapid, 1-day prediction & 0.22 & - & ultra-rapid observed versus predicted \\
IGS final, observed & 0.03 & - & Ray et al. (2017) \\
\hline
\end{tabular}


Table 2 Navigation messages including ERP information

\begin{tabular}{|c|c|c|c|}
\hline System & Signal & ERPs & Reference \\
\hline GPS CNAV & L2C, L5 & message type 32 & $\begin{array}{l}\text { IS-GPS-200M } \\
\quad(2021), \text { IS-GPS- } \\
\text { 705H (2021) }\end{array}$ \\
\hline GPS CNAV-2 & $\mathrm{L} 1 \mathrm{C}$ & subframe 3 page 2 & IS-GPS-800H (2021) \\
\hline BDS CNAV-1 & $\mathrm{B} 1 \mathrm{C}$ & subframe 3 page 3 & CSNO (2017a) \\
\hline BDS CNAV-2 & $\mathrm{B} 2 \mathrm{a}$ & message type 32 & CSNO (2017b) \\
\hline BDS CNAV-3 & $\mathrm{B} 2 \mathrm{~b}$ & message type 30 & CSNO (2020) \\
\hline QZSS CNAV & L2C, L5 & message type 32 & $\begin{array}{l}\text { IS-QZSS-PNT-004 } \\
\text { (2021) }\end{array}$ \\
\hline QZSS CNAV-2 & $\mathrm{L} 1 \mathrm{C}$ & subframe 3 page 2 & $\begin{array}{l}\text { IS-QZSS-PNT-004 } \\
\text { (2021) }\end{array}$ \\
\hline IRNSS NAV & L5, S & message type 11 & ISRO (2017) \\
\hline
\end{tabular}

of a given constellation in the RINEX 4.00 navigation data format. Likewise, the present analysis is confined to comparing ERPs of the individual constellations without distinguishing the individual navigation message types.

In April 2014, a pre-operational GPS CNAV transmission started with a subset of satellites. Since February 2015, GPS CNAV is transmitted by all Block IIR-M and IIF satellites (Steigenberger et al. 2015). GPS CNAV is also transmitted by the latest generation of GPS III satellites launched since December 2018. It is emphasized, though, that the transmission of GPS CNAV is currently declared as pre-operational and "and should not be used for safety-of-life or other critical purposes until the government declares them operational." (https://www.gps.gov/systems/gps/modernization/cnav/).

According to IS-GPS-200K (2019), GPS CNAV polar motion and polar motion rates already include the diurnal and semidiurnal tides, $\triangle \mathrm{UT} 1$ and $\Delta \mathrm{UT} 1$, in addition, include contributions of zonal tides. The same is stated for QZSS and IRNSS (see references in Table 2), whereas no information on this topic is given for BDS.

User algorithms as provided in the various ICDs specify that the pole coordinates and $\Delta \mathrm{UT} 1$ at an epoch $t$ in the vicinity of the reference epoch $t_{\mathrm{ERP}}$ are computed as

$$
\begin{aligned}
& x_{p}(t)=x_{p}\left(t_{\mathrm{ERP}}\right)+\dot{x}_{p}\left(t_{\mathrm{ERP}}\right) \cdot \Delta t \\
& y_{p}(t)=y_{p}\left(t_{\mathrm{ERP}}\right)+\dot{y}_{p}\left(t_{\mathrm{ERP}}\right) \cdot \Delta t \\
& \Delta \mathrm{UT} 1(t)=\Delta \mathrm{UT} 1\left(t_{\mathrm{ERP}}\right)+\Delta \mathrm{UT} 1\left(t_{\mathrm{ERP}}\right) \cdot \Delta t
\end{aligned}
$$

with

$\Delta t=t-t_{\mathrm{ERP}}$ where both $t$ and $t_{\mathrm{ERP}}$ are referred to as the constellationspecific system time. None of the constellations foresees an explicit consideration of periodic corrections to this linear model, which naturally limits the quality of this model.

Updated GPS ERP data sets are currently transmitted twice per day. The earliest transmit time $t_{t m}$ of the first set is between midnight and 03:00, but most records are transmitted before $02: 00$. For the second data set, the transmit time is between $22: 15$ and midnight. The reference epoch of the ERPs $t_{\text {ERP }}$ varies between 16:38-17:04 and 19:50-19:57 in a regular pattern and is typically 62 to $70 \mathrm{~h}$ ahead of the first transmit time. $\mathrm{t}_{\mathrm{ERP}}$ of the morning ERP values differs by roughly one day from the evening ERP values. However, the polar motion values of these two epochs agree within $\pm 1 \mu$ as when propagated to the same epoch with the corresponding polar motion rates (Eq. 5). The $\Delta \mathrm{UT} 1$ values agree within $\pm 50 \mathrm{~ns}$. Furthermore, the polar motion rates and the $\Delta \mathrm{UT} 1$ values of the morning and evening epochs are identical, also indicating that the ERP data of the evening transmission are based on prestored onboard data and do not reflect new information from an intermediate ground upload. Therefore, only the morning ERP values are considered in the following analysis.

For GPS, only ERP data after June 15, 2020, are considered in the present analysis due to inconsistent ERP data transmission schemes applied across the GPS constellation prior to that epoch. In early 2020, GPS III satellites already used a transmission scheme similar to the current one with $\mathrm{t}_{\mathrm{ERP}} 40$ to $70 \mathrm{~h}$ ahead of transmit time. For Block IIR-M and IIF satellites, $t_{\mathrm{ERP}}$ and $\mathrm{t}_{\mathrm{tm}}$ differed in general only by 10 to $30 \mathrm{~min}$, but few records also reached differences of up to $10 \mathrm{~h}$.

For completeness, we note that the specification of ERP data in GPS CNAV and CNAV-2 message was revised in mid 2020. Starting with IS-GPS200L (2020), IS-GPS-705G (2020), and IS-GPS-800G (2020) $\Delta$ UT1 $=$ UT1 - UTC has been replaced by $\Delta \mathrm{UT} 1_{\mathrm{GPS}}=\mathrm{UT} 1-\mathrm{GPST}$. In contrast to UTC, GPS time (GPST) is a continuous time scale and avoids potential problems in the vicinity of leap second changes. However, this change has not become effective yet, and the transmitted ERP data are based on the earlier specifications for the entire analysis period of our study.

According to NGA (2020), the National Geospatial-Intelligence Agency (NGA) provides ERPs to the GPS control segment that are based on data of the United States Naval Observatory (USNO). Based on the comparison with the broadcast ERPs, it can be concluded that the NGA data provide the current source of CNAV ERPs. The NGA parameters and hence the transmitted CNAV data are updated on a daily basis. The NGA predictions for polar motion and $\Delta \mathrm{UT} 1$ at epoch $t$ are described by a simple model with offset, drift, and harmonic terms: 
$\mathrm{P}(t)=\mathrm{O}_{\mathrm{P}}+\mathrm{D}_{\mathrm{P}} \cdot \Delta t_{p}+\sum_{i=2}^{3} \mathrm{~S}_{\mathrm{P}, i} \cdot \sin \varphi_{\mathrm{P}, i}+\mathrm{C}_{\mathrm{P}, i} \cdot \cos \varphi_{\mathrm{P}, i}$

$\Delta \mathrm{UT} 1(t)=\mathrm{O}_{\mathrm{U}}+\mathrm{D}_{\mathrm{U}} \cdot \Delta t_{u}+\sum_{i=1}^{2} \mathrm{~S}_{\mathrm{U}, i} \cdot \sin \varphi_{\mathrm{U}, i}+\mathrm{C}_{\mathrm{U}, i} \cdot \cos \varphi_{\mathrm{U}, i}$

with

$\varphi_{\mathrm{P}, i}=\frac{2 \pi \Delta t_{p}}{p_{i}}, \quad \varphi_{\mathrm{U}, i}=\frac{2 \pi \Delta t_{u}}{p_{i}}, \quad \Delta t_{p}=t-t_{p}, \quad \Delta t_{u}=t-t_{u}$

and the polar motion index P: $x_{p}$ or $y_{p}$, the $\Delta \mathrm{UT} 1$ index $\mathrm{U}$, the offset coefficient $\mathrm{O}$, the drift coefficient $\mathrm{D}$, the reference epoch for polar motion $t_{p}$, the reference epoch for $\Delta \mathrm{UT} 1 t_{u}$, the semiannual period $p_{1}(182.625 \mathrm{~d})$, the annual period $p_{2}$ ( $365.25 \mathrm{~d})$, the Chandler period $p_{3}(435 \mathrm{~d})$, the annual sine/ cosine polar motion terms $\mathrm{S}_{\mathrm{P}, 2}$ and $\mathrm{C}_{\mathrm{P}, 2}$, the Chandler sine/ cosine polar motion terms $\mathrm{S}_{\mathrm{P}, 3}$ and $\mathrm{C}_{\mathrm{P}, 3}$, the semiannual sine/ cosine $\Delta \mathrm{UT} 1$ terms $\mathrm{S}_{\mathrm{U}, 1}$ and $\mathrm{C}_{\mathrm{U}, 1}$, fixed to $\mathrm{S}_{\mathrm{U}, 1}=-0.006 \mathrm{~s}$ and $\mathrm{C}_{\mathrm{U}, 1}=-0.007 \mathrm{~s}$, the annual sine/cosine $\Delta \mathrm{UT} 1$ terms $\mathrm{S}_{\mathrm{U}, 2}$ and $\mathrm{C}_{\mathrm{U}, 2}$, fixed to $\mathrm{S}_{\mathrm{U}, 2}=-0.022 \mathrm{~s}$ and $\mathrm{C}_{\mathrm{U}, 2}=0.012 \mathrm{~s}$.

For $\Delta \mathrm{UT1}$, the harmonic terms are fixed to the values given above, i.e., only offset and drift are estimated model parameters, whereas six independent parameters represent polar motion. The time interval of observations contributing to the estimation of this model is unknown. NGA publishes the model coefficients as well as tabulated ERP predictions based on Eq. 7/8 and includes zonal tides and subdaily terms at its ftp server (see data availability statement) in two different versions. The predicted ERPs cover seven days and 180 days, respectively. The latter interval might be driven by the requirement to maintain navigation function for 180 days without ground contact (AUTONAV, Menn and Bernstein 1994). As the ERPs published by NGA do not contain rates, these seem to be derived from the tabulated predictions. Based on inspection of the respective data, it may be inferred that a difference quotient covering the first three days of predicted NGA ERPs is used to obtain the CNAV ERP rates.
New BDS ERP records are transmitted once per day with updates at about $00: 35$ or 01:00. The immediately preceding midnight epoch is usually adopted as $\mathrm{t}_{\mathrm{ERP}}$. The comparison with IGS or IERS ERP series suggests that the BDS broadcast ERPs are also derived from a harmonic prediction model as used by NGA. Unlike GPS, which uses new predictions every day, the BDS prediction model is updated irregularly at intervals of $4-20$ days. This resulted in larger errors and pronounced discontinuities of the transmitted ERPs at the model update epochs.

In contrast to the other systems, new QZSS ERP records are transmitted once every hour. The first transmission usually takes place at 3 min after the full hour. $t_{\mathrm{ERP}}$ refers to the next full hour, i.e., $57 \mathrm{~min}$ after $\mathrm{t}_{\mathrm{tm}}$. According to IS-QZSSPNT-004 (2021), the QZSS ERPs have a fit interval of two hours and a validity interval of $12 \mathrm{~h}$.

New IRNSS ERP records are transmitted once per day with updates between midnight and 0:03. $t_{\mathrm{ERP}}$ always refers to the corresponding midnight epoch. Between November 20,2020 , and January 21, 2021, no updates of the underlying predictions were performed for polar motion data transmitted by IRNSS. Accordingly, the pole misclosures that will be introduced later are zero during that time period. Therefore, this time interval is excluded from the further analysis of the IRNSS ERPs. Unfortunately, no details about the origin of the ERP data and the parameterization of the ERP prediction model are provided for BDS, QZSS, and IRNSS.

In order to better understand the transmission scheme of the broadcast ERPs and to identify their zero age of data, the best agreement with the IERS C04 series (Bizouard et al. 2018) was identified by propagating the broadcast ERPs to different trial epochs $t_{0}$. The resulting zero-age-of-data epochs

$t_{0}=\left\lfloor t_{\mathrm{tm}}\right\rfloor-d t$

provided in Table 3 are expressed by their offset $d t$ from the midnight epoch preceding the epoch of first transmission of an ERP data set. In addition, it is evaluated if the application of the IERS2010 subdaily ERP model improves the RMS values or not. The broadcast ERP values are propagated from
Table 3 Best agreement of broadcast ERP values with the IERS C04 reference series at zero-age of data

\begin{tabular}{|c|c|c|c|c|c|c|}
\hline \multirow[t]{2}{*}{ System } & \multirow[t]{2}{*}{$\mathrm{Sd}$} & \multirow{2}{*}{$\begin{array}{l}\text { X-pole } \\
\mathrm{RMS}_{\text {min }}[\mathrm{mas}]\end{array}$} & \multicolumn{2}{|l|}{ Y-pole } & \multicolumn{2}{|l|}{$\Delta \mathrm{UT} 1$} \\
\hline & & & $\mathrm{RMS}_{\min }[\mathrm{mas}]$ & $d t_{\mathrm{PM}}[\mathrm{h}]$ & $\mathrm{RMS}_{\min }[\mathrm{ms}]$ & $d t_{\mathrm{UT}}[\mathrm{h}]$ \\
\hline GPS & $\mathrm{x}$ & 0.15 & 0.13 & 24 & 0.09 & 0 \\
\hline BDS & $\mathrm{x}$ & 0.48 & 0.36 & 36 & 0.18 & 17 \\
\hline QZSS & & 0.13 & 0.12 & 16 & 0.08 & 20 \\
\hline IRNSS & & 0.30 & 0.26 & 33 & 0.11 & 4 \\
\hline
\end{tabular}

The column $s d$ indicates if subdaily ERP corrections have been applied. $d t$ stands for the offset w.r.t. the midnight epoch of the day of transmission to minimize the difference to the reference series (Eq. 9) 
$t_{\mathrm{ERP}}$ to $t_{0}$ according to (5) and the subdaily ERPs are optionally removed at $t_{0}$ as the reference series is free of subdaily ERP variations.

For BDS, only the first epoch of an updated ERP data set is used as the following epochs are strongly affected by prediction errors. These epochs have been determined by analyzing the discontinuities in polar motion and $\triangle \mathrm{UT} 1$ time series w.r.t. a reference series as shown in Fig. 6b. As a consequence, only $11 \%$ of the available BDS ERP data are used for this analysis.

For GPS, significantly different values for $d t$ are obtained for polar motion and $\Delta \mathrm{UT} 1$. Whereas a shift of one day shows the lowest polar motion RMS values of about 0.15 mas, no shift is necessary for $\Delta \mathrm{UT} 1$. Correction for subdaily polar motion improves the RMS values by a factor of more than two, whereas the improvement for $\Delta \mathrm{UT} 1$ is only minor. Compared to GPS, the RMS values for BDS are larger by a factor of three for polar motion and a factor of two for $\triangle \mathrm{UT1}$. Like for GPS, a significant improvement could be achieved when applying the subdaily ERP model, thus demonstrating that the broadcast ERPs of both constellations include the subdaily tides at the zero age of data epoch, rather than those at the ERP epoch or the first transmit epoch.

The RMS values of QZSS are similar to those of GPS, but the best agreement is achieved without correcting for the subdaily ERP model. This is surprising as the spectra of the QZSS ERPs contain significant peaks at the typical subdaily periods. However, as the amplitudes of the subdaily terms included in the QZSS ERPs are smaller than the IERS model, the smallest RMS values are obtained without considering the subdaily ERP model. The latter is also true for IRNSS, but the RMS with respect to the reference series is worse by a factor of more than two for polar motion.

The ICDs of all considered systems state that subdaily corrections are included in the broadcast ERPs, but do not specify how exactly the subdaily tides are incorporated into the ERP values and rates at the ERP epoch. In fact, our analysis suggests that subdaily tides are applied in the ERP values at the zero age of data epoch for GPS and BDS, while IRNSS ERPs appear to be free of these corrections. In view of a linear propagation over several hours or even days, including periodic subdaily variations is questionable. For proper handling, the subdaily ERP model would have to be subtracted from the GPS and BDS ERPs at the zero age of data epoch and added back after propagation to the desired epoch with the ERP rates. However, as the errors of the broadcast ERPs at transmission time are dominated by the prediction errors of the ERPs, the error contribution of the subdaily ERPs is essentially negligible in the current context. The same is true for the linear propagation of $\Delta \mathrm{UT} 1$, neglecting the reduction to UT1R. The latter introduces an RMS error of $10 \mu$ s with peak values of $50 \mu$ s.

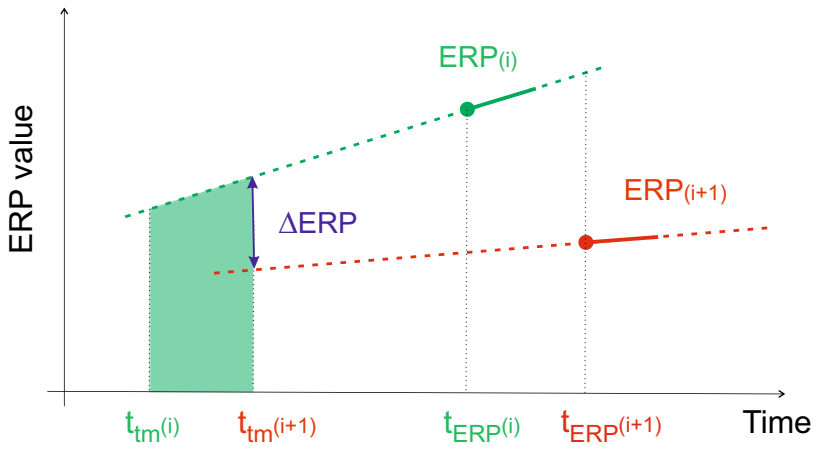

Fig. 5 ERP misclosure $\Delta$ ERP with $t_{\text {tm }}$ : transmission time, $t_{\mathrm{ERP}}$ : ERP reference epoch. The shaded area marks the interval during which $\mathrm{ERP}_{i}$ should be applied by the user. Dashed lines describe the linear ERP model defined by ERP values and rates at the ERP epoch $t_{\text {ERP }}$

Table 4 Misclosures of broadcast ERP data

\begin{tabular}{lllll}
\hline System & $\begin{array}{l}\text { Update inter- } \\
\text { val }\end{array}$ & $\begin{array}{l}\text { X-pole } \\
{[\mathrm{mas}]}\end{array}$ & $\begin{array}{l}\text { Y-pole } \\
{[\mathrm{mas}]}\end{array}$ & $\begin{array}{l}\Delta \mathrm{UT1} \\
{[\mathrm{ms}]}\end{array}$ \\
\hline GPS & $1 \mathrm{~d}$ & 0.61 & 0.41 & 0.17 \\
BDS & $4-20 \mathrm{~d}$ & 1.24 & 0.73 & 0.32 \\
QZSS (1 h) & $1 \mathrm{~h}$ & 0.05 & 0.05 & 0.02 \\
QZSS (1 d) & & 0.61 & 0.51 & 0.45 \\
IRNSS & $1 \mathrm{~d}$ & 0.67 & 0.54 & 0.18 \\
\hline
\end{tabular}

\section{Broadcast ERP quality}

The precision of the broadcast ERPs is evaluated by misclosures of consecutive data sets, whereas comparisons with an external reference series assess the accuracy.

\section{Internal consistency}

The internal consistency of independent ERP estimates can be evaluated by ERP misclosures as illustrated in Fig. 5 . The ERP values are evaluated at consecutive $t_{\mathrm{tm}}$ epochs $i$ and $i+1$ with their rates, and the differences $\triangle \mathrm{ERP}$ at these epochs allow to assess the internal consistency. $\mathrm{t}_{\mathrm{tm}(i)}$ is the epoch when a new set $\operatorname{ERP}(i)$ is transmitted the first time. The users apply these ERPs until transmission of the next data set $\operatorname{ERP}(i+1)$ at $\mathrm{t}_{\mathrm{tm}(i+1)}$ illustrated by the shaded area in Fig. 5.

The RMS values of the ERP misclosures are given in Table 4. For GPS and IRNSS, the consistency is at roughly 0.5 mas for polar motion and $0.17 \mathrm{~ms}$ for $\Delta \mathrm{UT} 1$. When looking at the hourly update rate of QZSS, the misclosures are smaller by a factor of ten for polar motion and seven for $\Delta \mathrm{UT} 1$. However, when looking at daily intervals, polar 

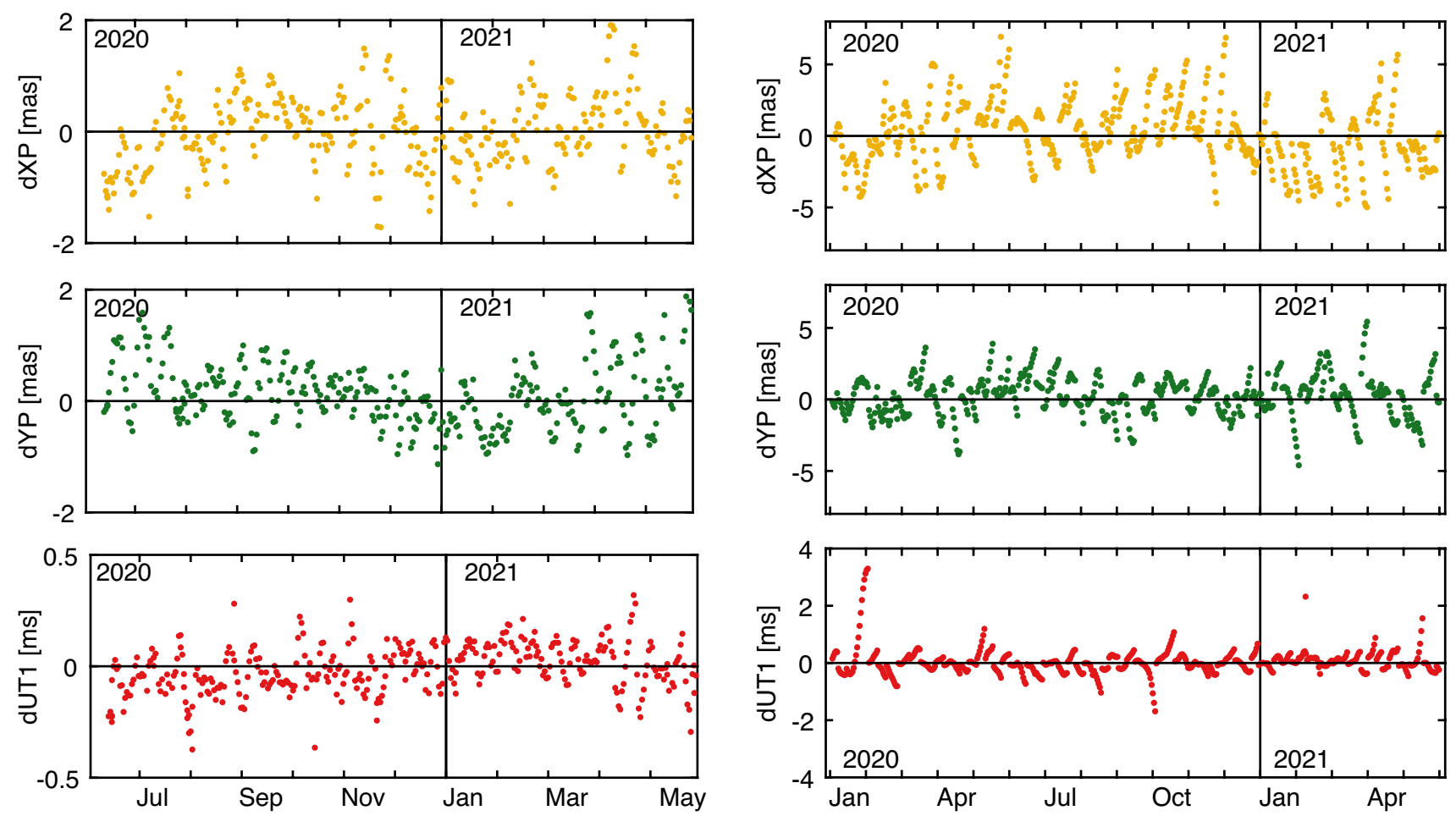

(a) GPS

(b) BDS
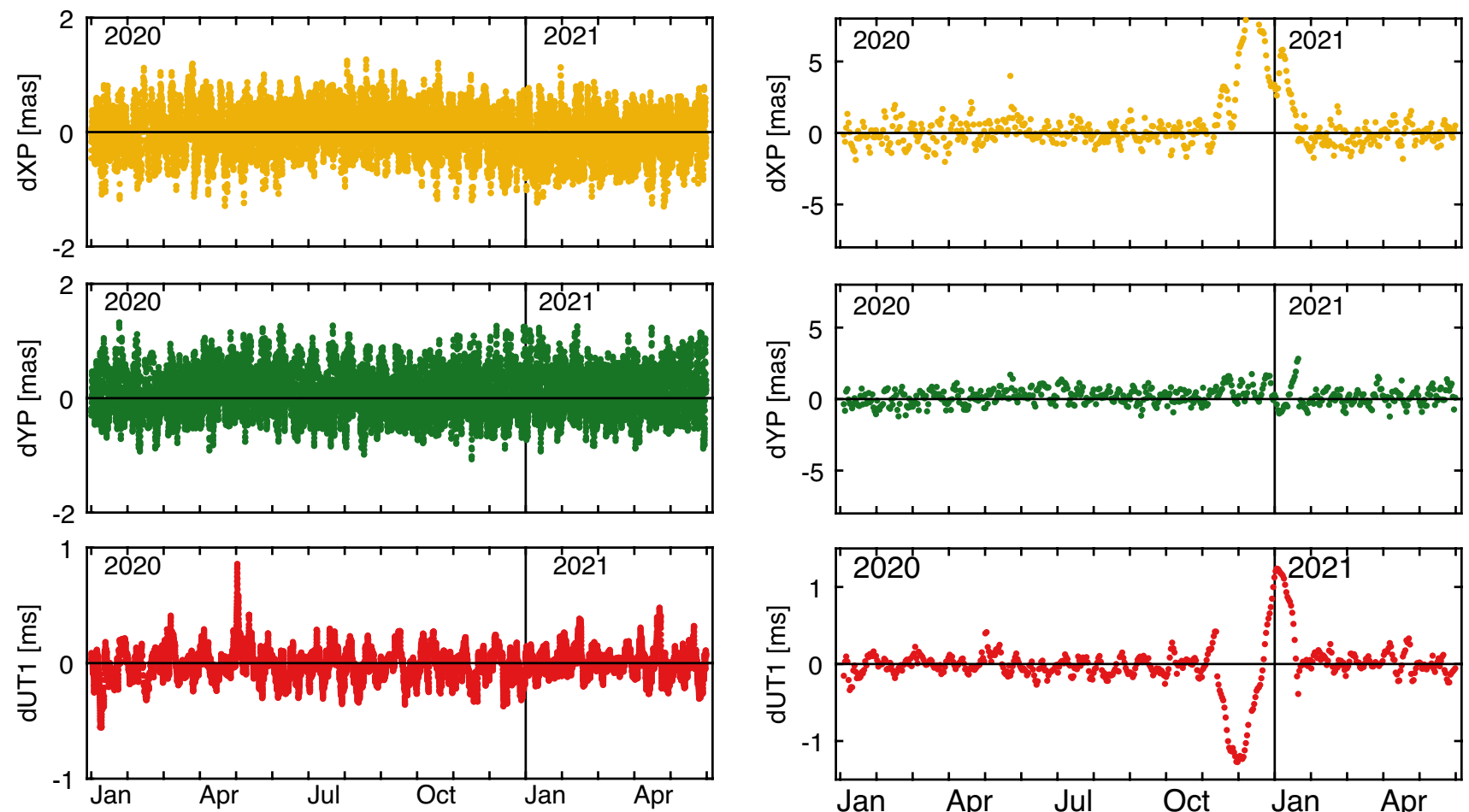

(c) QZSS

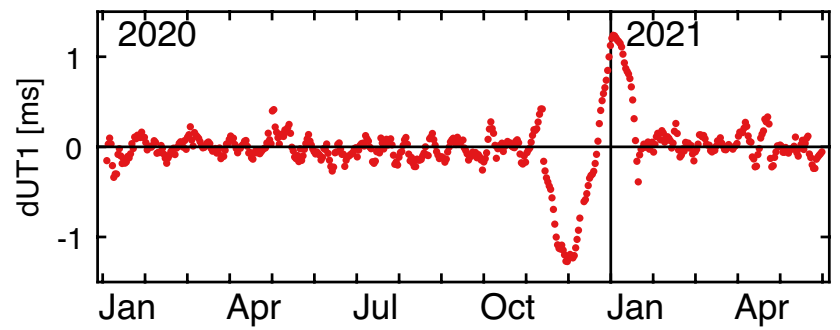

(d) IRNSS

Fig. 6 Differences between broadcast ERP records at $t_{\mathrm{tm}}$ and the IERS C04 ERP series. Please note the different scales of the individual plots 
motion is at a similar level as GPS and $\triangle \mathrm{UT} 1$ is worse by a factor of more than two. Whereas the pattern of the misclosures is pretty random for GPS, QZSS, and IRNSS, the situation is different for BDS. Most of the misclosures are within $\pm 10 \mu$ as for polar motion $(87 \%)$ and $\pm 10 \mu \mathrm{s}$ for $\Delta \mathrm{UT} 1(84 \%)$. The remaining misclosures reach up to 8 mas for polar motion and $3 \mathrm{~ms}$ for $\Delta \mathrm{UT} 1$, thus causing the large misclosure values in Table 4 . This behavior can be explained by the 4-20 day update interval of the underlying ERP forecast model for BDS, which results in very small discontinuities during transmission of the same model but large discontinuities when switching to the next parameter set.

\section{Comparison with reference series}

The IERS C04 ERP series consistent with ITRF2014 (Bizouard et al. 2018) is used as an external reference to evaluate the broadcast ERP quality. In the case of BeiDou, raw CNAV-1/2/3 ERP messages with void data, i.e., a null bit pattern, have been discarded in the data collection. Furthermore, occasional cases of erroneous data transmissions (from satellites $\mathrm{C} 45 / \mathrm{C} 46$ ) resulting in grossly wrong ERP values have been identified and rejected in a coarse outlier screening. Unless otherwise noted, the comparison of $\mathrm{C} 04$ and broadcast ERPs was performed at first transmit time of the individual broadcast ERP records. At each epoch, subdaily ERP corrections were added to C04 ERP values according to IERS conventions, whereas no corrections were applied to the broadcast ERPs as specified in the constellation-specific ICDs (see Table 2).

The ERP difference time series are shown in Fig. 6. None of the series shows a significant bias w.r.t. the C04 reference series, neither for polar motion nor for $\triangle \mathrm{UT} 1$. The scatter of the GPS and QZSS ERP differences is quite random. GPS has maximum differences of \pm 2 mas for polar motion and $\pm 0.5 \mathrm{~ms}$ for $\Delta \mathrm{UT}$. For BDS, steadily increasing differences with peak values of 7 mas and
$3 \mathrm{~ms}$ are visible during time intervals of $4-20$ days due to less frequent ERP model updates already mentioned above. The anomalous ERP values of IRNSS in December 2020 and January 2021 are included in Fig. 6d. They reach a maximum of 10 mas for $x_{p}$ and $\pm 1.2 \mathrm{~ms}$ for $\Delta \mathrm{UT} 1$ and are caused by missing updates of the IRNSS broadcast ERP model during that time period indicated by zero polar motion misclosures and $\Delta \mathrm{UT} 1$ misclosures smaller than $\pm 10 \mu \mathrm{s}$.

Numerical values of the RMS differences of the broadcast ERPs w.r.t. the reference series are given in Table 5. The RMS values are evaluated for three different epoch types: the transmission time and the next transmission time resulting in best and worst case values as well as for a time series with 15 min sampling to evaluate the average user performance. Due to the frequent updates, QZSS shows the smallest RMS values of about 0.4 mas for polar motion as well as the smallest differences for the three cases. The reason for the better agreement for a longer prediction time $\left(t_{i+1}\right)$ is unknown. GPS and IRNSS have a very similar performance that is worse by a factor of roughly two compared to QZSS. For $\triangle \mathrm{UT1}$, GPS, QZSS and IRNSS show the same level of RMS differences of 0.1 to $0.2 \mathrm{~ms}$, but BDS is worse by a factor of three. The same comparisons were also done with the IGS final ERP series (IGS 2021b) but are not shown here as they are very similar to the $\mathrm{C} 04$ comparison differing by only up to $10 \mu$ as for polar motion and $20 \mu$ s for $\Delta \mathrm{UT} 1$.

Compared to Table 3 , the values in Table 4 are larger by a factor of up to eight, whereas the $\Delta \mathrm{UT} 1$ differences are significantly smaller. Overall, the broadcast ERP performance experienced by GNSS users appears to be mainly driven by the time between the zero age of data epoch $t_{0}$ and the time of first transmission, $\mathrm{t}_{\mathrm{tm}}$. This is particularly obvious for BDS, which uses less-frequent forecast model updates than other constellations. Furthermore, it may be noted that the forecast errors dominate the contributions of subdaily ERP variations and mask a possible neglect or ICD-related uncertainties in this correction.

Table 5 RMS differences of broadcast ERPs with respect to IERS C04. Differences are evaluated at transmission time (best case, column $t_{i}$ ), one epoch before transmission time (worst case, column $t_{i-1}$ ) and for a time series at $15 \mathrm{~min}$ intervals (average case, column $t s$ )

\begin{tabular}{|c|c|c|c|c|c|c|c|c|c|c|}
\hline \multirow[t]{2}{*}{ System } & \multirow{2}{*}{$\begin{array}{l}\text { Update } \\
\text { interval }\end{array}$} & \multicolumn{3}{|c|}{ X-pole [mas] } & \multicolumn{3}{|c|}{ Y-pole [mas] } & \multicolumn{3}{|c|}{$\Delta \mathrm{UT} 1[\mathrm{~ms}]$} \\
\hline & & $\mathrm{t}_{i}$ & ts & $\mathrm{t}_{i-1}$ & $\mathrm{t}_{i}$ & ts & $\mathrm{t}_{i-1}$ & $\mathrm{t}_{i}$ & ts & $\mathrm{t}_{i-1}$ \\
\hline GPS & $1 \mathrm{~d}$ & 0.65 & 0.96 & 1.13 & 0.60 & 0.64 & 0.88 & 0.11 & 0.15 & 0.19 \\
\hline BDS & $4-20 \mathrm{~d}$ & 2.27 & 2.41 & 2.56 & 1.42 & 1.48 & 1.55 & 0.55 & 0.51 & 0.55 \\
\hline QZSS & $1 \mathrm{~h}$ & 0.39 & 0.39 & 0.34 & 0.39 & 0.30 & 0.28 & 0.13 & 0.14 & 0.14 \\
\hline IRNSS & $1 \mathrm{~d}$ & 0.75 & 0.96 & 1.12 & 0.56 & 0.67 & 0.76 & 0.11 & 0.14 & 0.15 \\
\hline
\end{tabular}

IRNSS RMS values for ts considering also the anomalous data from November 2020 until January 2021 amount to 2.19 and 0.74 mas for polar motion and $0.36 \mathrm{~ms}$ for $\Delta \mathrm{UT} 1$ 


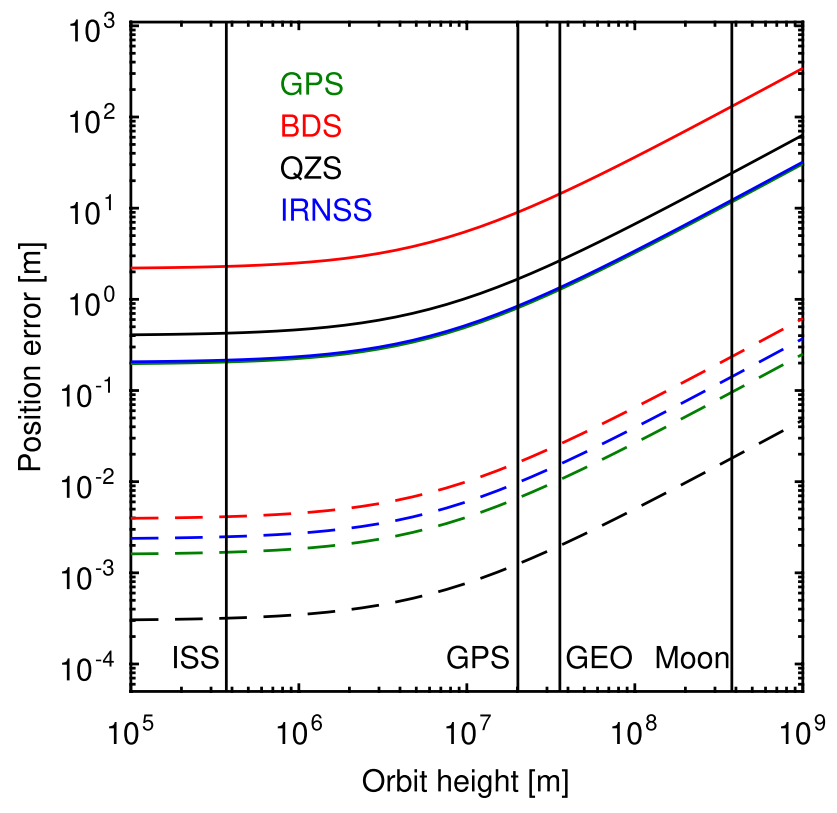

Fig. 7 ERP-induced position errors for different orbit heights for broadcast ERPs evaluated at $t_{\mathrm{tm}}$ : minimum values (dashed line) and maximum values (solid line). Selected orbit heights are indicated by vertical lines: International Space Station (ISS) at low earth orbit, GPS satellites at medium earth orbit, geostationary satellites (GEO), and the moon

\section{Conclusions}

Even though the availability of ERPs might be of interest for real-time precise point positioning (PPP) to properly model site displacement due to pole tides, their primary use is certainly in the field of space-borne navigation. Possible applications include navigation of satellites in low earth or geostationary orbit (Capuano et al. 2017), as well as navigation to the moon (Delépaut et al. 2020). Figure 7 illustrates the impact of ERP-induced errors on the transformation for ECEF to ECI for different orbit heights. Minimum and maximum errors evaluated at $t_{\mathrm{tm}}$ from the analysis interval are given. With a single-frequency COTS GPS receiver connected to a signal simulator, Capuano et al. (2017) obtained a single epoch positioning error in GEO of about $17 \mathrm{~m}$. As the ERP-induced position error at GEO is well below one meter for GPS, the broadcast ERP quality is certainly compatible with current performance needs in this application.

The same is true for onboard orbit determination of the Chinese geostationary satellite TJS-2 presented by Wang et al. (2021). The GNSS receiver onboard TJS-2 is capable of tracking GPS, GLONASS and BDS-2 singlefrequency code observations. For the transformation to the inertial frame, predicted Bulletin-A ERPs are used. Wang et al. (2021) report a 3D position difference RMS value of $21.3 \mathrm{~m}$ between the onboard and a postprocessed solution using the same observation data but more sophisticated models as well as IGS final orbit and clock products. However, Capuano et al. (2017) could also demonstrate a positioning error of less than one meter for simulated Galileo dual-frequency observations. For this use case, the broadcast ERPs would significantly contribute to the position error in the inertial frame.

ERPs included in the broadcast messages of GPS, BDS, QZSS, and IRNSS allow for ECEF/ECI transformations and vice versa onboard GNSS-equipped spacecraft without the need for external data. For GPS, BDS, and QZSS, the current ERP accuracy appears sufficient in view of the overall positioning accuracy achievable in GNSS-based navigation. Due to the less frequent ERP model update intervals, the BeiDou ERPs show a reduced accuracy.

A conceptual problem in the use of broadcast ERPs results from the unclear, and most likely misleading, specifications for applications of subdaily tides in the ICDs of all GNSS presently providing ERP data as part of their navigation messages. While errors resulting from the associated uncertainties are generally smaller than the contribution of ERP forecast errors, it would be strongly advisable to transmit only mean ERP values and rates that do not include any subdaily tidal contributions. This would ensure consistency with other geodetic services, provide better transparency, and facilitate the handling of broadcast ERPs on the user side. With respect to $\triangle \mathrm{UT} 1$, improved predictions could potentially be achieved when replacing UT1 with reduced UT1R. This is particularly true for GPS, where UT1 rates are based on a linear difference quotient approximation of predicted values over a 3-day interval and certainly affected by the zonal tides with periods of 5 days and up. However further analysis would be required to trade the possible accuracy gain against the increased complexity of evaluating the UT1 - UT1R model at the user end.

Finally, we recall that the European Galileo system does not, so far, provide ERPs as part of its INAV and FNAV navigation messages. This contrasts sharply with the benefit that Galileo tracking provides for space-borne users in view of its superior broadcast ephemeris accuracy (Montenbruck et al. 2022) and the upcoming introduction of a dedicated high-accuracy service (Fernandez-Hernandez et al. 2018). The addition of ERP data to the current navigation messages should therefore be investigated and is strongly encouraged.

Acknowledgements We'd like to thank our station operators R. Botha (Hartebeesthoek Radio Astronomy Observatory, South Africa), J. Cheong (University of New South Wales, Australia), A. Hauschild (DLR, Germany), M. Kriegel (DLR, Germany), R. Langley (University of New Brunswick, Canada), D. O'Gara (University of Hawaii, USA), Y. Verschelle (Université de la Polynésie Française, French Polynesia), and B. Zimmerman (University of Texas at Austin, USA) for their long-standing support. 
Funding Open Access funding enabled and organized by Projekt DEAL.

Data Availability Bulletin A is available at https://www.iers.org/Share dDocs/News/EN/BulletinA.html; IERS C04 is available at https://www. iers.org/IERS/EN/DataProducts/EarthOrientationData/eop.html; IGS ultra-rapid and final ERP files are available at ftp://igs.bkg.bund.de/ IGS/products/orbit; NGA ERP data are available at ftp://ftp.nga.mil/ pub2/gps/eopp36/. The datasets generated during the current study are available from the corresponding author on reasonable request.

Open Access This article is licensed under a Creative Commons Attribution 4.0 International License, which permits use, sharing, adaptation, distribution and reproduction in any medium or format, as long as you give appropriate credit to the original author(s) and the source, provide a link to the Creative Commons licence, and indicate if changes were made. The images or other third party material in this article are included in the article's Creative Commons licence, unless indicated otherwise in a credit line to the material. If material is not included in the article's Creative Commons licence and your intended use is not permitted by statutory regulation or exceeds the permitted use, you will need to obtain permission directly from the copyright holder. To view a copy of this licence, visit http://creativecommons.org/licenses/by/4.0/.

\section{References}

Bar-Sever YE, Bertiger WI (2012) Method and apparatus for autonomous, in-receiver prediction of GNSS ephemerides. Patent US $8,120,529$ B2

Bizouard C, Lambert S, Gattano C, Becker O, Richard JY (2018) The IERS EOP 14C04 solution for Earth orientation parameters consistent with ITRF 2014. J Geod 93(5):621-633. https://doi.org/10. 1007/s00190-018-1186-3

CSNO (2017a) BeiDou Navigation Satellite System Signal In Space Interface Control Document Open Service Signal B1C (Version 1.0). URL http://en.beidou.gov.cn/SYSTEMS/Officialdocument/ 201806/P020180608525871869457.pdf

CSNO (2017b) BeiDou Navigation Satellite System Signal In Space Interface Control Document Open Service Signal B2a (Version 1.0). URL http://www.beidou.gov.cn/xt/gfxz/201712/P020171226 742357364174.pdf

CSNO (2020) BeiDou Navigation Satellite System Signal In Space Interface Control Document: Open Service Signal B2b (Version 1.0). URL http://www.beidou.gov.cn/xt/gfxz/202008/P020200803 362059116442.pdf

Capitaine N (1986) The Earth rotation parameters: conceptional and conventional definitions. Astron Astrophys 162(1-2):323-329

Capuano V, Shehaj E, Blunt P, Botteron C, Farine PA (2017) High accuracy GNSS based navigation in GEO. Acta Astronaut 136:332-341. https://doi.org/10.1016/j.actaastro.2017.03.014

Dehant V, Mathews PM (2015) Precession, nutation and wobble of the earth. Cambridge University Press

Delépaut A, Giordano P, Ventura-Traveset J, Blonski D, Schönfeldt M, Schoonejans P, Aziz S, Walker R (2020) Use of GNSS for lunar missions and plans for lunar in-orbit development. Adv Space Res 66(12):2739-2756. https://doi.org/10.1016/j.asr.2020.05.018

Fernandez-Hernandez I, Vecchione G, Diaz-Pulido F, Jeannot M, Valentaite G, Blasi R, Reyes J, Simon J (2018) Galileo high accuracy: A program and policy perspective. In: Proc 69th International Astronautical Congress, IAC 18-B2.4.1

Gambis D (2006) DORIS and the determination of the Earth's polar motion. J Geod 80(8-11):640-656. https://doi.org/10.1007/ s00190-006-0043-y
IGS (2021a) GNSS combined ultra-rapid solution Earth rotation parameters product. https://doi.org/10.5067/GNSS/GNSS IGSUE RP_001

IGS (2021b) GNSS final weekly combined Earth rotation parameters product. https://doi.org/10.5067/GNSS/GNSS_IGSERPP_001

IS-GPS-200K (2019) Interface Specification IS-GPS-200: Navstar GPS Space Segment/Navigation User Segment Interfaces. URL https:// www.gps.gov/technical/icwg/IS-GPS-200K.pdf

IS-GPS-200L (2020) Interface Specification IS-GPS-200: Navstar GPS Space Segment/Navigation User Segment Interfaces. URL https:// www.gps.gov/technical/icwg/IS-GPS-200L.pdf

IS-GPS-200M (2021) Interface Specification IS-GPS-200: Navstar GPS Space Segment/Navigation User Segment Interfaces. URL https://www.gps.gov/technical/icwg/IS-GPS-200M.pdf

IS-GPS-705G (2020) Navstar GPS Space Segment/User Segment L5 Interfaces. Tech. rep., Global Positioning System Directorate Systems Engineering and Integration, URL https://www.gps.gov/ technical/icwg/IS-GPS-705G.pdf

IS-GPS-705H (2021) Navstar GPS Space Segment/User Segment L5 Interfaces. Tech. rep., Global Positioning System Directorate Systems Engineering and Integration, URL https://www.gps.gov/ technical/icwg/IS-GPS-705H.pdf

IS-GPS-800G (2020) Navstar GPS Space Segment/User Segment L1C Interfaces. Tech. rep., Global Positioning System Directorate Systems Engineering and Integration, URL http://www.gps.gov/techn ical/icwg/IS-GPS-800G.pdf

IS-GPS-800H (2021) Navstar GPS Space Segment/User Segment L1C Interfaces. Tech. rep., Global Positioning System Directorate Systems Engineering and Integration, URL http://www.gps.gov/techn ical/icwg/IS-GPS-800H.pdf

IS-QZSS-PNT-004 (2021) Quasi-zenith satellite system interface specification satellite positioning, navigation and timing service. Tech. rep., Cabinet Office, URL http://qzss.go.jp/en/technical/download/ pdf/ps-is-qzss/is-qzss-pnt-004.pdf

ISRO (2017) Indian Regional Navigation Satellite System Signal in Space ICD for Standard Positioning Service, Version 1.1. Tech. Rep. IRSO-IRNSS-ICD-SPS-1.1, ISRO Satellite Centre, Indian Space Research Organization, Bangalore

International GNSS Service (IGS), RINEX Working Group and Radio Technical Commission for Maritime Services Special Committee 104 (RTCM-SC104) (2021) RINEX, The Receiver Independent Exchange Format, Version 4.00

Johnston G, Riddell A, Hausler G (2017) The International GNSS Service. In: Teunissen P, Montenbruck O (eds) Springer Handbook of Global Navigation Satellite Systems. Springer, pp 967-982. https://doi.org/10.1007/978-3-319-42928-1_33

Lieske JH, Lederle T, Fricke W, Morando B (1977) Expressions for the precession quantities based upon the IAU (1976) system of astronomical constants. Astron Astrophys 58:1-16

McCarthy DD (1996) IERS Conventions (1996). IERS Technical Note 21, US Naval Observatory

Menn M, Bernstein H (1994) Ephemeris observability issues in the Global Positioning System (GPS) autonomous navigation (AUTONAV). In: Proceedings of 1994 IEEE Position, Location and Navigation Symposium - PLANS'94, IEEE, https://doi.org/10.1109/ plans.1994.303376

Montenbruck O, Hauschild A, Hessels U (2011) Characterization of GPS/GIOVE sensor stations in the CONGO network. GPS Solut 15(3):193-205. https://doi.org/10.1007/s10291-010-0182-8

Montenbruck O, Kunzi F, Hauschild A (2022) Performance assessment of GNSS-based real-time navigation for the Sentinel-6 spacecraft. GPS Solut 26:12. https://doi.org/10.1007/s10291-021-01198-9

Montenbruck O, Steigenberger P, Aicher M (2020) A long-term broadcast ephemeris model for extended operation of GNSS satellites. NAVIGATION 68(1):199-215. https://doi.org/10. 1002/navi.404 
NGA (2020) NGA GNSS division earth orientation. Tech. rep., URL ftp://ftp.nga.mil/pub2/gandg/website/gnss/data/prod_docs/ NGAGNSSEOPPParameters.pdf

Nothnagel A, Artz T, Behrend D, Malkin Z (2016) International VLBI Service for Geodesy and Astrometry. J Geod 91(7):711-721. https://doi.org/10.1007/s00190-016-0950-5

Pearlman MR, Noll CE, Pavlis EC, Lemoine FG, Combrink L, Degnan JJ, Kirchner G, Schreiber U (2019) The ILRS: approaching 20 years and planning for the future. J Geod 93(11):2161-2180. https://doi.org/10.1007/s00190-019-01241-1

Petit G, Luzum B (2010) IERS Conventions (2010). IERS Technical Note 36, Verlag des Bundesamtes für Kartographie und Geodäsie, Frankfurt am Main

Ramatschi M, Bradke M, Nischan T, Männel B (2019) GNSS data of the global GFZ tracking network. https://doi.org/10.5880/GFZ.1. 1.2020 .001

Ray J, Rebischung P, Griffiths J (2017) IGS polar motion measurement accuracy. Geod Geodyn 8(6):413-420. https://doi.org/10.1016/j. geog.2017.01.008

Rothacher M, Beutler G, Herring T, Weber R (1999) Estimation of nutation using the Global Positioning System. J Geophys Res 104(B3):4835-4859. https://doi.org/10.1029/1998JB900078

Schuh H, Behrend D (2012) VLBI: a fascinating technique for geodesy and astrometry. J Geodyn 61:68-80. https://doi.org/10.1016/j.jog. 2012.07.007

Seidelmann PK (1982) 1980 IAU theory of nutation: the final report of the IAU working group on nutation. Celest Mech 27(1):79-106. https://doi.org/10.1007/bf01228952

Steigenberger P, Montenbruck O, Hessels U (2015) Performance evaluation of the early CNAV navigation message. NAVIGATION 62(3):219-228. https://doi.org/10.1002/navi.111

Torge W, Müller J (2012) Geodesy, 4th edn. deGruyter. https://doi.org/ $10.1515 / 9783110250008$

Wallace P, Capitaine N (2006) Precession-nutation procedures consistent with IAU 2006 resolutions. Astro Astrophys 459:981-985. https://doi.org/10.1051/0004-6361:20065897

Wang M, Shan T, Li M, Liu L, Tao R (2021) GNSS-based orbit determination method and flight performance for geostationary satellites. J Geod. https://doi.org/10.1007/s00190-021-01545-1

Willis P, Lemoine FG, Moreaux G, Soudarin L, Ferrage P, Ries J, Otten M, Saunier J, Noll C, Biancale R, Luzum B (2015) The international DORIS service (IDS): Recent developments in preparation for ITRF2013. In: International Association of Geodesy Symposia, Springer International Publishing, pp 631-640, https://doi. org/10.1007/1345_2015_164

Wooden W, Gambis D (2004) Explanatory supplement to IERS bulletins A and B. Tech. rep., IERS Rapid Service/Prediction Centre;
IERS Earth Orientation Centre, URL https://hpiers.obspm.fr/ eoppc/bul/bulb/explanatory.html

Yoder CF, Williams JG, Parke ME (1981) Tidal variations of Earth rotation. J Geophys Res 86(B2):881-891. https://doi.org/10.1029/ jb086ib02p00881

Publisher's Note Springer Nature remains neutral with regard to jurisdictional claims in published maps and institutional affiliations.

Peter Steigenberger received his master and $\mathrm{PhD}$ degree in Geodesy from Technische Universität München (TUM) in 2002 and 2009, respectively. Currently, he is a senior researcher at DLR's German Space Operations Center (GSOC). His research interests focus on GNSS data analysis, in particular the precise orbit and clock determination of GNSS satellites and the evolving navigation systems Galileo, BeiDou, and QZSS.

Oliver Montenbruck is head of the GNSS Technology and Navigation Group at DLR's German Space Operations Center, where he started work as a flight dynamics analyst in 1987. His current research activities comprise space-borne GNSS receiver technology, autonomous navigation systems, spacecraft formation flying, precise orbit determination, and multi-constellation GNSS.

Markus Bradke received his master degree in Geoinformatics and Geodesy from Hochschule Neubrandenburg in 2012. He is a Development and Operations Engineer for GNSS applications at the German Research Centre for Geosciences (GFZ) and is currently leading the Operational Data Centre. Furthermore, he is the chair of the Infrastructure Committee of the International GNSS Service (IGS).

Markus Ramatschi received his master and $\mathrm{PhD}$ degree in Geophysics from Technische Universität Clausthal (TUC) in 1992 and 1998, respectively. He is a scientist at the German Research Centre for Geosciences (GFZ) since 2000, and he is operating a globally distributed network of GNSS receiving stations.

Uwe Hessels got his diploma in electrical engineering in $1982 . \mathrm{He}$ started at BKG (formerly IfAG) with the mobile laser ranging project MTLRS-1 in Wegener Medlas and other campaigns. Since 1995, he has been a member of the GNSS operations center of the Geodetic Observatory Wettzell (GOW), with currently about 20 worldwide GNSS sites. 